\title{
REPORT
}

\section{Epidemiology, diagnostic possibilities, and treatment of tuberculosis}

\author{
R Kurth, W H Haas
}

Ann Rheum Dis 2002;61(Suppl II):ii59-ii61

\section{EPIDEMIOLOGY}

On a worldwide scale, infectious diseases still account for about $25 \%$ of all deaths, only surpassed by cardiovascular diseases. ${ }^{1}$ The picture, however, is dissimilar when industrialised and developing countries are compared. In Germany, for example, only $1 \%$ of all deaths are due to infectious diseases, whereas in developing countries-for example, in subSaharan Africa $49 \%$ of all deaths are due to infections. Mycobacterium tuberculosis is the second biggest killer world wide, with only HIV/AIDS responsible for more deaths. Tuberculosis notification rates in the year 2000 in many developing countries reached 100 or more per 100000 population. ${ }^{2}$ A relatively high rate of underreporting has to be assumed for many countries, and estimates reach as high as more than 500 new infections per 100000 people. In general, tuberculosis is a disease of the poor: the less developed health systems are, the higher the rates of new cases of tuberculosis (fig 1).
One third of the world population has been infected by $M$ tuberculosis. From this pool, almost nine million acute cases developed in 2000, leading to two million deaths. Twenty two high burden countries in the developing areas of the world carry about $80 \%$ of the disease burden. Owing to HIV/AIDS, we see, especially in high burden countries in sub-Saharan Africa, an increase of up to $10 \%$ of new tuberculosis infections a year. More than $95 \%$ of all cases and $98 \%$ of all deaths occur in developing countries.

In the European region, tuberculosis notification rates per 100000 population are the highest in Russia and the other successor states to the Soviet Union. ${ }^{3}$ Prisons in Russia are a particular focus of tuberculosis. ${ }^{4}$ There are about 1.1 million prisoners in Russia, of whom $10-20 \%$ are estimated to be infected. This high rate is in part due to the breakdown of the public health system in the former Soviet Union in the 1990s, but also to a certain degree to the rapid increase of HIV/AIDS,

\section{Tuberculosis notification rates, 2000}

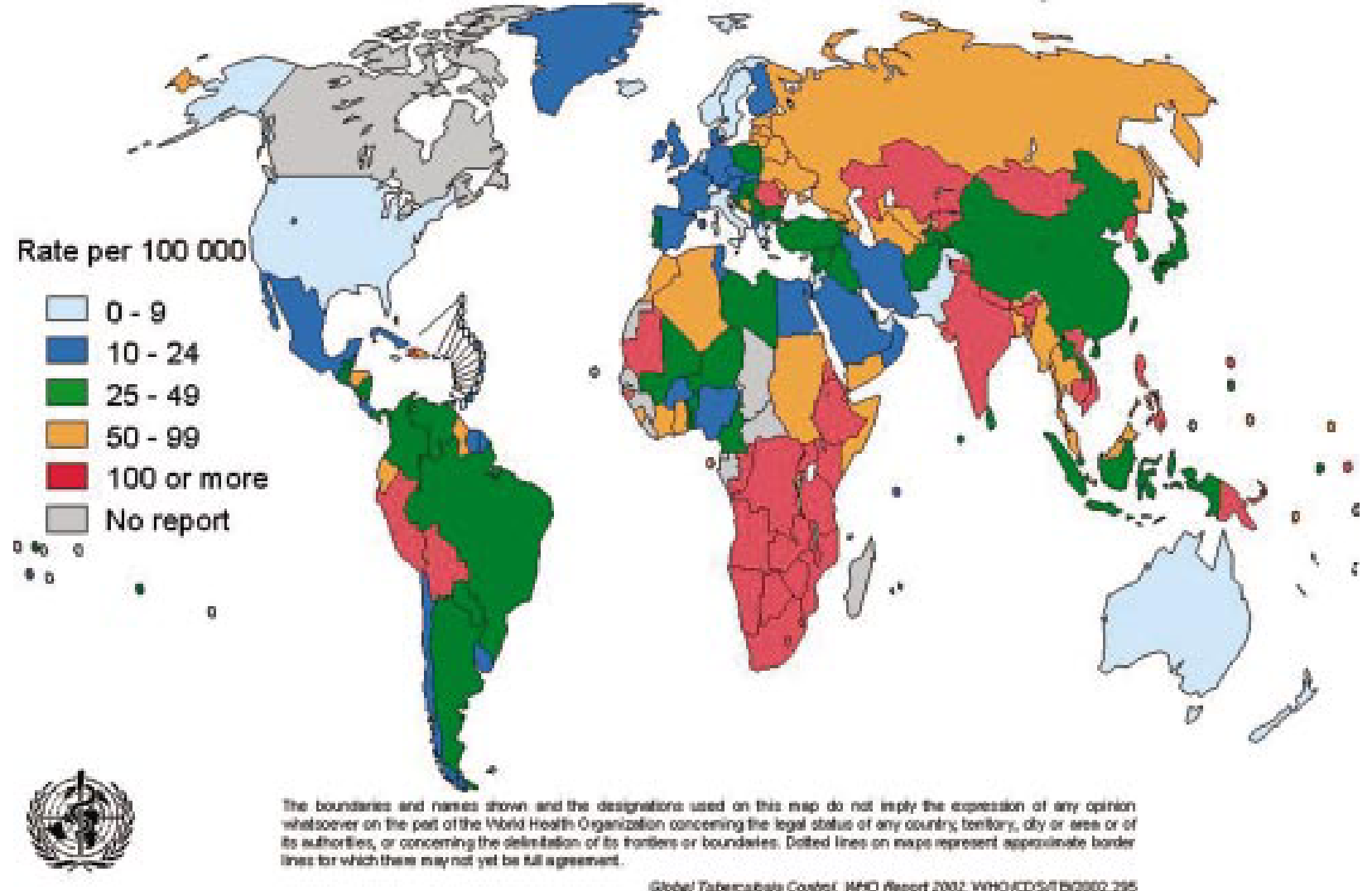

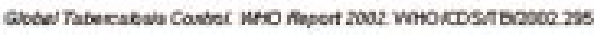

Figure 1 Tuberculosis notification rates, 2000. Reproduced, with permission, from reference 2. 
Box 1 Amplification techniques for direct detection of mycobacteria

- Nucleic acid amplification methods

- Polymerase chain reaction

- Transcription mediated amplification

- Strand displacement amplification

- Ligase chain reaction

- Target sequences for amplification

- "Single copy" genes-for example, 16S rDNA

- Repetitive DNA

which allows $M$ tuberculosis to infect and cause clinical disease more readily. ${ }^{5}$ In Eastern Europe, especially, we can also quantify a steep increase in some of the successor states of the former Soviet Union. In contrast, the incidence of tuberculosis in Western Europe is slowly decreasing, delayed, however, by the relatively high degree of infection in migrants and refugees to those countries. For Germany, it is estimated that about $30 \%$ of all new cases occur in the foreign-born population. ${ }^{6}$

We have seen in Germany a steady decline in the incidence of tuberculosis over the past 50 years, already beginning at a time when antibiotics were not yet available. The decrease in incidence equals about one case per 100000 population a year. The incidence of tuberculosis in Germany is now at about nine cases per 100000 population. In 2001, almost 8000 new cases were reported for a population of about 83 million German citizens.

Grouping the cases of tuberculosis by age, it easily becomes evident that tuberculosis in Germany is a disease of the elderly population. The observed incidence in people above 69 years is 4-8 times higher than the average incidence in adults younger than 30. In old age, the efficacy of the defence by the immune system declines, allowing, in some cases, old contained infections to reappear.

The mode of transmission of tuberculosis is well knownnamely, human to human by inhalation of aerosols from infected subjects. Children are rarely transmitters, as their bacterial load is usually low. Furthermore, cavernae are rarely seen in children early in infection and their coughs are weaker than in adults. ${ }^{7}$ Positive acid fast microscopy of sputum is indicative of highly infectious subjects.

\section{DIAGNOSIS OF TUBERCULOSIS}

The clinical presentation of the patient may be very variable, but the anamnestic situation is already indicative for risks. People born outside Germany and those from lower social classes are at a higher risk for being infected and developing disease. Routine laboratory tests are of little relevance for diagnosis. Acid fast microscopy of the sputum is a rapid and reliable tool, but does not differentiate between $M$ tuberculosis and non-tuberculous mycobacteria. Sputum is used for both solid and liquid culture assays. Subsequent species identification, today, is performed by molecular or other rapid techniques (for example, high performance liquid chromatography). ${ }^{8}$ Nucleic acid analysis requires amplification of individual gene segments-for example, the 16S rDNA, or probing genes by hybridisation. Molecular detection and characterisation is performed by employing a variety of methods of genome amplification (box 1 ).

Radiological imaging is still of prime value. Chest $x$ ray examinations should always be included in the diagnostic investigation of suspected cases of tuberculosis. Computed tomography or nuclear magnetic resonance are usually reserved for providing reliable images of severe pathological consequences and complications of infection (for example, tuberculous meningitis, tuberculoma). Ultrasound may also

\section{DNA target sequences}

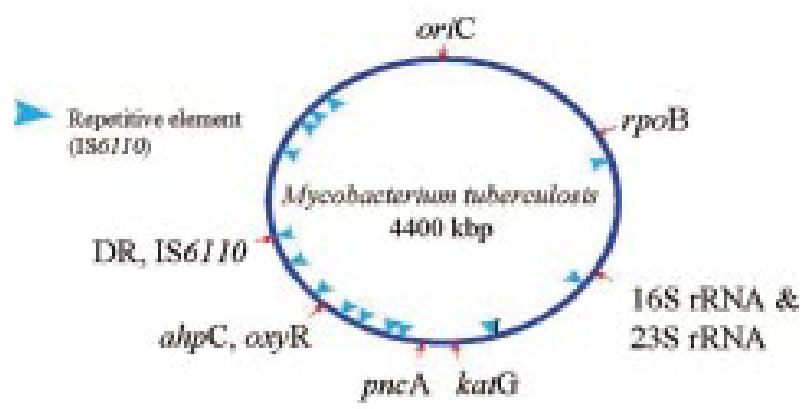

Figure 2 Schematic representation of the Mycobacterium tuberculosis $\mathrm{H} 37 \mathrm{Rv}$ genome. Location of target sequences for amplification indicated.

be very helpful in diagnosing extrapulmonary disease. Bronchoscopy should always be performed in the case of compression of the tracheobronchial tree or endobronchial tuberculosis, when it may be diagnostic and therapeutic at the same time. However, bronchoalveolar lavage has no higher sensitivity for isolation of the organism than sputum examination

The previous use of solid culture media required incubation periods of up to 12 weeks. The inoculum had to contain at least 1000 bacteria. Once growth of bacteria could be observed, subculture was required, necessitating another one to two weeks before biochemical analysis could be performed. The use of liquid culture media is much faster (one to two weeks). Growth in liquid culture systems can be detected by $\mathrm{CO}_{2}$ production, oxygen consumption, or other sensitive detection methods. ${ }^{10}$ The nucleic acid amplification methods, already mentioned above, vary and may detect DNA or RNA sequences (box 1). Target sequences for amplification include single copy genes and repetitive DNA (fig 2).

The sensitivity of polymerase chain reaction for direct detection of tuberculosis as compared with culture in a specimen negative by smear microscopy rarely exceeds $80 \%$, whereas specificity is desirably high $(97 \%)$. The positive predictive value, in other words the percentage of true cases tested positive, depends very much on the frequency of active disease in the population tested and may be very low, especially in countries with a low incidence.

A variety of target sequences have been described for direct detection and molecular characterisation of $M$ tuberculosis. A selection is displayed in fig 2 . The rpoB gene, if amplifiable with specific primers, is diagnostic for the infection by tuberculosis, and sequence analysis may also indicate resistance to rifampicin. ${ }^{11}$ Other target genes can be used to learn more about additional antibiotic resistance and clonal relationship of multidrug resistant bacterial strains.

\section{THERAPEUTIC ASPECTS}

Isoniazid, rifampicin, pyrazinamide, and ethambutol are the first line drugs used in Germany for the directly observed treatment of short course (six months) or DOTS, recommended by WHO. ${ }^{12}$ When this treatment is started in Germany, bacterial cultures are usually obtained to determine whether drug resistant tuberculosis strains are present. A strain is classed as resistant when $1 \%$ of all bacterial cells have a clearly reduced sensitivity to the drugs tested. Under special circumstances direct analysis of mutations in the rpoB gene can be done by polymerase chain reaction and direct sequencing of the amplified product (for example, at the National Reference Centre in Germany), as resistance to rifampicin most often indicates multidrug resistance. 
Chemoprophylaxis with isoniazid is recommended in Germany for purified protein derivative (PPD) negative children exposed to infectious subjects. In children who have a positive PPD skin test and no signs or symptoms of disease indicative of latent infection, preventive chemotherapy with isoniazid has to be started and maintained for nine months. ${ }^{12}$ If drug resistance is suspected isoniazid may be replaced by rifampicin.

Future recommendations for adults in Germany aim at strengthening the role of preventive chemotherapy after individual assessment of the risk of reactivation compared with the untoward effects and efficacy of the intervention (Schaberg T, Centre for Pneumology, Diakoniekrankenhaus, Rotenburg, Germany, personal communication). In patients treated with tumour necrosis factor $\alpha$ inhibitors the risk of reactivation and severe disease is very high, as this treatment knocks out a central pathway in the defence of tuberculosis. Therefore, thorough diagnostic investigation and early preventive treatment are recommended before starting antitumour necrosis factor $\alpha$ treatment (urgent safety restriction of the corresponding German National Control Authority, the Paul Ehrlich Institute, Langen, Germany, 2000). The use of preventive chemotherapy as a general epidemiological tool is complicated by the proportion of infected subjects who carry potentially drug resistant strains, which is already high and increasing in Europe.
Authors' affiliations

R Kurth, W H Haas, Robert Koch-Institute, Nordufer 20, 13353 Berlin, Germany

Correspondence to: Dr R Kurth; KurthR@rki.de

\section{REFERENCES}

1 WHO. Removing obstacles to healthy development. WHO Report 1999. (WHO/CDS/99.1.)

2 WHO. Global tuberculosis control: surveillance, planning, financing WHO Report 2002. (WHO/CDS/TB/2002.295.)

3 EuroTB (InVS/KNCV), and the national coordinators for tuberculosis surveillance in the WHO European Region. Surveillance of tuberculosis in Europe. Report on tuberculosis cases notified in 1999. EuroTB 2002.

4 Perelman MI. Tuberculosis in Russia. Int J Tuberc Lung Dis 2000; 4:1097-103.

5 Barnes PF, Lakey DL, Burman WJ. Tuberculosis in patients with HIV infection. Infect Dis Clin North Am 2002;16:107-26.

6 Deutsches Zentralkomitee zur Bekämpfung der Tuberkulose. 27. Informationsbericht. Frankfurt a. Main: pmi Verlag, 2002.

7 Correa AG. Unique aspects of tuberculosis in the pediatric population. Correa AG. Unique aspects of tuber
Clin Chest Med 1997; 18:89-98.

8 Hale YM, Pfyffer GE, Salfinger M. Laboratory diagnosis of mycobacterial infections: new tools and lessons learned. Clin Infect Dis 2001;33:834-46.

9 Perkins MD. New diagnostic tools for tuberculosis. Int J Tuberc Lung Dis 2000; 4(suppl 2):S182-8.

10 Schluger NW. Changing approaches to the diagnosis of tuberculosis. Am J Respir Crit Care Med 2001;164:2020-4.

11 Pfyffer GE. Drug-resistant tuberculosis: resistance mechanisms and rapid susceptibility testing. Schweiz Med Wochenschr 2000:130:1909-13.

2 Schaberg T, Forssbohm M, Hauer B, Kirsten D, Kropp R, Loddenkempe $R$, et al. Guidelines for drug treatment of tuberculosis in adults and $\mathrm{R}$, et al. Guidelines for drug treatment of tube
childhood. Pneumologie 2001;55:494-511. 